\title{
Portable NMR in food analysis
}

\begin{abstract}
Low-field ${ }^{1} \mathrm{H}$ NMR relaxometry is an important tool used to investigate on the most abundant components of intact foodstuffs based on relaxation parameters and amplitude of the NMR signals. In particular, information on water compartments, diffusion and movement can be obtained by detecting proton signals prevalently ascribable to $\mathrm{H}_{2} \mathrm{O}$ contained in foodstuffs. The main advantage of this technique is that it does not require any pretreatment of the sample and once developed, standard protocols based on rapid measurements can be easily transferred to quality control applications. An actual breakthrough for the low-field NMR application to food science has certainly been the development of unilateral NMR sensors. These devices present an attractive option for non-invasive assessment of compositional and microstructure of food materials. They are portable and allow easy sample access, which makes them attractive for quality control in industrial environments and directly on sealed packaged foods. A price to pay is the inhomogeneity of the magnetic field, which compromises the sensitivity of these devices. However, recent developments in unilateral magnets design offer larger sensitive volume, higher sensitivity and shorter dead times. In this brief review, we highlight the different uses of unilateral NMR devices in food analysis.
\end{abstract}

Keywords: Portable NMR, Food science, Relaxation times, Water mobility, Self-diffusion coefficient, Quality control process

\section{Background}

Pulsed low-resolution NMR is an appreciated and powerful technique in food science and technology. NMR instruments operating in the time domain (TD) at low field are widely used for investigating on relationships between food properties and its composition and structure. In fact foodstuffs are structurally and chemically heterogeneous systems whose constituents, due to the different molecular mobility, show different relaxation and diffusive properties. Pulsed low-resolution NMR technique is suitable to study the dynamic properties of matter like self-diffusion, flow and relaxation. The two major NMR relaxation phenomena are longitudinal relaxation characterized by a relaxation time $T_{1}$, and transverse relaxation, characterized by $T_{2}$.

Hahn echo decay experiment can be used to measure the transverse relaxation time. The amplitude of the echo is recorded for different spacings of the two pulses. Echo amplitude is influenced by molecular diffusion (Brownian

\footnotetext{
*Correspondence: noemi.proietti@cnr.it

1 Istituto di Metodologie Chimiche, Laboratorio di Risonanza Magnetica,

"Annalaura Segre", CNR, Monterotondo, 00015 Rome, Italy

Full list of author information is available at the end of the article
}

motion) because of the fluctuations of the local magnetic field. Thus, the amplitude of the echo at the time $t$ is related to the transverse relaxation $T_{2}$, gradient $G$, selfdiffusion coefficient $D$, gyromagnetic ratio $\gamma$, and the half echo spacing $\tau$ through the following equation:

$$
M_{t}=\exp \left(-\frac{1}{T_{2}}-\frac{\gamma^{2} G^{2} D \tau^{2} t}{3}\right)
$$

In the case of most conventional NMR magnets, the field inhomogeneity is sufficiently small that at short $\tau$ values the second term of the equation becomes negligible even for faster diffusing molecules such as water. Instead, in the case of a strong inhomogeneous magnetic field the second term of Eq. (1) cannot be neglected.

The pulsed field gradient NMR method is one of the main NMR methods for obtaining dynamic information, such as the self-diffusion coefficient. Self-diffusion data provide detailed information about molecular organization and phase structure. Self-diffusion rates are quite sensitive to structural changes and to binding and association phenomena. Experimental self-diffusion values are directly related to molecular displacement. 
Many analytical protocols have been established for rapid and precise measurements of compositional properties [1-4]. These protocols are particularly appreciated in the food supply chain for quality and process control. In many applications, differences in self-diffusion coefficient and relaxation times have been exploited to differentiate different phases in food matrices [1-3]. For example, ${ }^{1} \mathrm{H}$ pulsed low-resolution NMR has been applied to study the effect of different physiological conditions on kiwifruit [5, 6], apple [7-10], carrot [11] and strawberry [3, 12].

The effect induced by ripening, drying, freezing, highpressure processing, bruising, browning, aging and osmotic drying has been studied [1, 2, 13-15].

The relationships between ${ }^{1} \mathrm{H}$ NMR relaxation data and some technological parameters of meat have been investigated [16-18].

NMR is a well-accepted analytical tool for evaluation of oil and moisture content in food $[1,19]$. The presence of non-freezable water, water mobility as well as the phase compositional and hydration phenomena have been investigated in many food matrices [1].

A breakthrough in the application of TD NMR to food science has been represented by the conceiving of compact and portable NMR sensors which enable the analysis of intact food matrices in a non-destructive, non-invasive way in situ. Sensors based on permanent magnets have been developed during the last years covering applications involving relaxometry, imaging and even spectroscopy with hand-held systems [20-23]. These devices require low maintenance costs and allow easy sample access, which makes them attractive for quality and process control in industrial environments.

\section{Portable single-sided NMR sensors with open geometry}

In conventional NMR instruments, the geometry of the magnet is referred to as closed geometry and the sample under study is placed in a homogeneous magnetic field. While this facilitates high signal-to-noise, geometrically correct, spatially resolved magnetic resonance imaging (MRI), it limits the range of samples that can be examined. In recent years, this limitation has been addressed by development of portable or unilateral NMR [22-24]. The geometry of portable NMR sensors, in which the object is exposed to the stray field of the magnet, is referred to as open geometry. In this case, the magnet may be placed to one side of the object fully preserving the integrity and the dimension of the sample under investigation and also allowing the measurement to whole packed products.

Nowadays, the unilateral NMR sensors available can be categorized into two classes. The first group operates in a strong magnetic field gradient [24], whereas the second one operates in region under more or less homogeneous magnetic field [22, 23]. Both approaches have their strengths and weaknesses.

Unilateral NMR developed by Blumich et al. [25] is characterized by a high magnetic field strength operating in the proton frequency range of $13-18 \mathrm{MHz}$ with a strong magnetic field gradient. This sensor requires very short (in the range of the microseconds) and very powerful RF pulses to achieve the desired frequency bandwidth. An example of such a sensor is sketched in Fig. 1a. Two antiparallel magnets are mounted on an iron yoke and the radio-frequency coil is positioned in the gap between the two magnets. The magnet may be designed either parallel or perpendicular to the sensor surface. Using this sensor, the depth of measurement into the investigated object may be varied by changing the RF coil [26]. The signal-to-noise ratio decreases by increasing the depth of measurement due to the $B_{1}$ stray field.

Another class of unilateral NMR instrument has been developed by Marble et al. [22, 27]. In these sensors, the unilateral magnet array consisted of three block magnets all magnetized along the same directions, see Fig. 1b. The sensitive spot (c) is located $\sim 1 \mathrm{~cm}$ above the face of the magnet. The magnet spacings are optimized to produce a locally homogeneous field in this region creating a relatively large MR sensitive volume above the surface RF coil (b). The resonance frequency of the NMR system is $4.68 \mathrm{MHz}$ and all pulse lengths are about of $8 \mu \mathrm{s}$ with $6 \mathrm{~dB}$ attenuation for the $90^{\circ}$ pulse and no attenuation for the $180^{\circ}$ pulse $[22,28]$.

Another portable NMR sensor with a novel one-sided access magnet design, termed NMR-MOLE (mobile lateral explorer), has been developed by Manz et al. [23]. This sensor is very efficient in terms of sensitivity and penetration depth. The magnet array is based on a barrel magnet operating at $3.3 \mathrm{MHz}$ with a central magnet positioned to provide a homogeneous region from 4 to $16 \mathrm{~mm}$ away from the probe, with maximum sensitivity at a depth of $10 \mathrm{~mm}$. Because of the lower diffusion attenuation in homogeneous field sensors, they are better suited for studying liquid samples such as aqueous solutions and biological tissue where unilateral or portable access is required.

A recent evolution of NMR sensors with an open geometry is the profile NMR MOUSE [29]. The magnet geometry consists of four permanent magnet blocks positioned on an iron yoke, see Fig. 1c. Two magnets are polarized along $y$ and two along $-y$. Magnets with the same polarization are separated by a small gap $d_{\mathrm{S}}$ whereas magnets with opposite polarization are separated by a gap $d_{\mathrm{B}}$. The sensor is placed on a lift which moves the sensitive volume constituted of a thin slice at a well-defined position through the object by varying the distance between the sensor and the object see Fig. 1d. The slice thickness depends on the experimental conditions and typically ranges between a few micrometers and $100 \mu \mathrm{m}$. The 
a
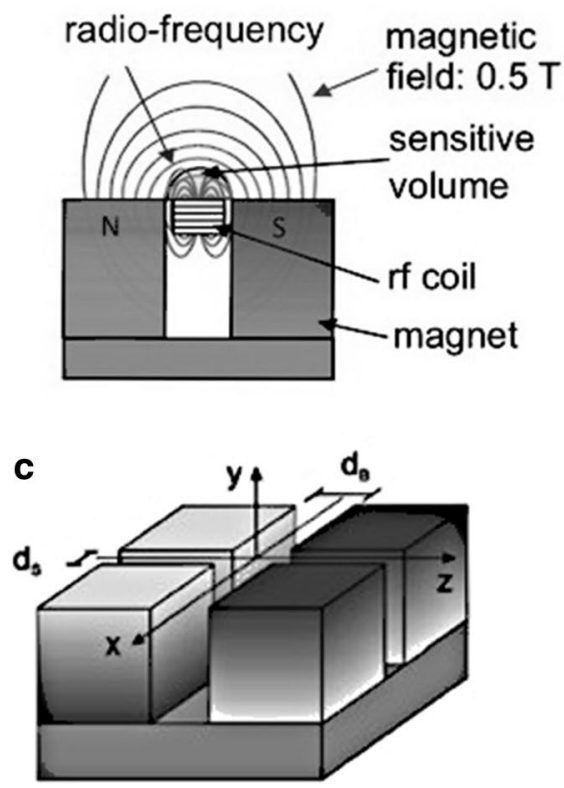

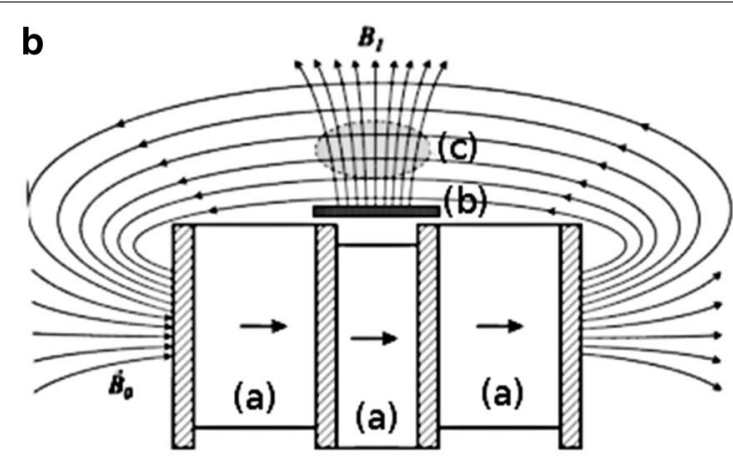

d

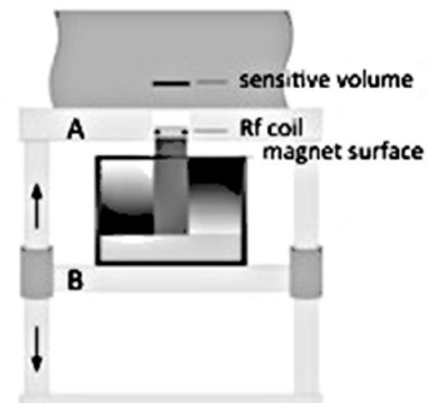

Fig. 1 a Skecth of a single-sided NMR sensor with open geometry (reproduced with permission from [25]). b Sketch of a single-sided magnet array with a sweet-spot (reproduced with permission from [41]). c Sketch of the geometry of the magnet of the profiler NMR MOUSE. d Sketch of the lift used to reposition the sensitive slice across the sample with a micrometric precision. The object is placed on top of the plate $A$, which is parallel to the movable plate $B$ where the magnet is mounted (reproduced with permission from [28])

magnetic field varies linearly with the space generating a constant magnetic field gradient of about $20 \mathrm{~T} / \mathrm{m}$ [29].

The amplitude values recorded by a modulated displacing of the sensor may provide a profile of proton spin densities as a function of the depth into the sample. However, not only the signal amplitude but also other parameters (i.e. relaxation times) can be reported against the depth scanned. Presently, a penetration depth up to $2.5 \mathrm{~cm}$ may be achieved with these sensors.

Due to strongly inhomogeneous magnetic field, the applications of portable NMR are limited as compared to those performed by benchtop instrumentation. FID cannot be detected, because NMR signal decays very quickly and must be recovered as an echo. The measurement of transverse relaxation time suffers for artifacts resulting from molecular diffusion in the strong magnetic field gradient. These artifacts are more pronounced in the case of small molecules that diffuse rapidly. In the presence of diffusion process, the transverse relaxation time values are shortened with respect to those measured in homogeneous field. Furthermore, the decay of the observed transverse magnetization in inhomogeneous field is a mixture of $T_{1}$ and $T_{2}$ and the early echoes oscillate in amplitude thus leading to an affective echo train decay time $T_{2 \text { eff }}$ instead only $T_{2}$ [30-32]. As well known, it is possible to reduce the effect of molecular diffusion on the
NMR signal collected in the magnetic field gradient by generating a series of spin echoes making the interpulse delay $\tau$ between $\pi / 2$ and $\pi$ pulses as short as possible.

Another limit in working with permanent magnets is represented by the temperature sensitivity. The NMR MOUSE is not temperature stabilized due to the high gradient of the sensor; a temperature change of the magnet causes a slight shift of the position of the sensitive volume [33]. Consequently, when working at room temperature, the applications are limited as compared to those performed by benchtop instrumentation and are mainly focused on the development of protocols applied in online inspections and for monitoring processes such as ripening, growth rate of fruits, drying process, and so on. Since $T_{2}$ tightly depends on temperature, in case of applications in an outdoor environment characterized by a temperature close to that of room temperature, a system to control the temperature during the measurements is strongly recommended to guarantee a good reproducibility. Anferova et al. [34] described two different types of mobile temperature control units operating with the NMR-MOUSE. They reported that in the case of elastomers, by the temperature stabilization the relative error of $T_{2}$ decreased by a factor of four on the short time scale. Without temperature stabilization, it may reach $30 \%$ in the long time range because of temperature changes in the laboratory. 


\section{Application of single-sided NMR sensors to food matrices}

Interesting applications of portable single-sided NMR sensors to food matrices have been reported in literature $[24,35]$. Despite the inhomogeneous field generated by these open geometry sensors, their potentiality for food analysis and control has been explored. An overview of food examined by unilateral devices is reported in Table 1. The advantage of this methodology is the possibility to analyze foods just placing the sensor on the food surface without needing to cut the sample into pieces suitable to fit into an NMR tube.

The determination of solid fat content (SFC) in food based on pulsed low-resolution NMR is an internationally accepted procedure [1]. Basically, the difference in transverse relaxation properties of solid and liquid fats was exploited to determine the solid and liquid contents. Although this technique is widely used even in industrial processes it cannot be applied on line.

Guthausen et al. investigated the applicability of a single-sided NMR sensor to measure the fat content in packaged food products [36, 37]. At this aim, two methods [35] were applied. One is based on differences in relaxation times which depend on molecular mobility and, consequently on the molecular structure (relaxation weighted method), whereas the other one exploits the fact that the diffusion coefficients of water and fat differ by more than an order of magnitude (diffusionweighted method). A pulse sequence was purposely developed for measuring fat and water content in packaged coffee creams. The packing material does not influence significantly the measure because it is relatively thin and the polymer material exhibits a short $T_{2 \text { eff }}$ relaxation time. The measurement time was about 1 min using the surface resonance frequency, whereas measurements at $3 \mathrm{~mm}$ depth resulted in considerable longer acquisition times (about $5 \mathrm{~min}$ ). The ratio of the final echoes and the first few echoes was the relevant NMR parameter, named NMR ratio. The NMR ratio exhibits a linear dependence with fat content (linear regression with a correlation coefficient of 0.996 ) thus indicating that a reasonable determination of the fat content is possible, see Fig. $2 \mathrm{a}$.

The relaxation method was used for calibrating the fat content in packaged mayonnaises and margarines. In fact, the relaxation time differences of fat and water protons may be exploited to generate a contrast by allowing a more accurate content determination. Again, a linear correlation was found between the fat percentage measured by NMR and the reference fat content, see Fig. 2b. In this case, data were analyzed by a chemometric approach obtaining a correlation coefficient of 0.991 . Such a linear correlation confirms the reliability of single-sided NMR for fat measurements even in packaged food. The chemometric approach was applied to the NMR signal which can be analyzed using supervised or unsupervised pattern recognition methods [38, 39]. These methods may be applied in food analysis for both quantitative and qualitative assessments. The knowledge of reference values allows for the calibration of the NMR response.

In another paper, Martini et al. [40] compared SFC determination obtained using conventional offline NMR instrument with determination obtained by single-sided NMR sensor. Authors explored the use of single-sided NMR to measure variation of SFC online during the crystallization of a fat product. Interesterified hydrogenated palm oil was added to canola oil in different proportions to obtain blends with different SFC values. Two different experiments were carried out to study whether the

Table 1 Reviews of portable NMR technologies and their applications in food science

\begin{tabular}{|c|c|c|c|}
\hline Reference & Food & Focus & Year \\
\hline Guthausen et al. [36] & Margarine; mayonnaise & Measurements of fat content & 2004 \\
\hline Guthausen et al. [37] & Coffee cream & Water moisture and fat analysis in packaged products & 2006 \\
\hline Martini et al. [40] & Vegetable oils & Solid fat content determination & 2005 \\
\hline Pedersen et al. [41] & Vegetable oils & Measurements of fat content & 2003 \\
\hline Haiduc et al. [42] & Fat emulsions & Microstructural quality of packaged food emulsion & 2007 \\
\hline Stork et al. [44] & Bottled beverages & Determination of dissolved oxygen in unopened table waters & 2001 \\
\hline Xu et al. [46] & Adulterated virgin olive oil & Detection of the adulteration of virgin olive oil in sealed bottles & 2014 \\
\hline Pinter et al. [48] & Tomato paste & Monitoring of spoilage in tomato paste processing industry & 2014 \\
\hline Veliyulin et al. [43] & Salmon & Fat content in live fish & 2005 \\
\hline Veliyulin et al. [28] & Dairy products & Measurements of fat content in packaged dairy products & 2008 \\
\hline Capitani et al. [49] & Kiwifruit & Water status as a function of season & 2010 \\
\hline Capitani et al. [50] & Kiwifruit & Monitoring of development and ripening & 2013 \\
\hline Capitani et al. [51] & Blueberry & Monitoring of shelf life & 2014 \\
\hline Adiletta et al. [52] & Pear & Monitoring of drying process & 2015 \\
\hline
\end{tabular}



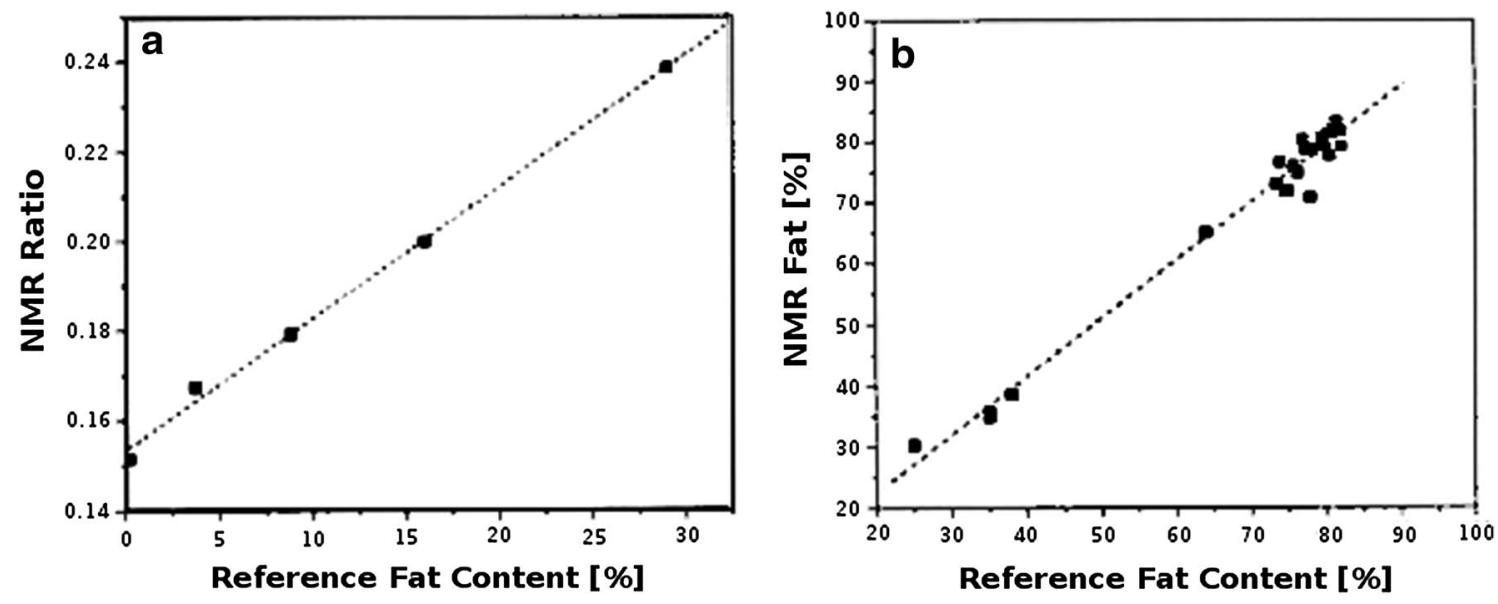

Fig. 2 a Correlation of the NMR ratio with the reference fat content of coffee creams. $\mathbf{b}$ Correlation diagram for measurements on mayonnaises and margarines with the reference fat content. Reproduced with permission from [34]

motion during the crystallization affected the measurements. In one experiment, agitation was stopped during the measurement and then restarted to let the crystallization continue until the next measurement. In the other experiments, measurements were carried out under agitation. To eliminate the temperature effect on measurements carried out by single-sided sensor, a proper correction was applied to the collected data. In the former case, the corrected data were close to those collected by a conventional offline NMR instrument particularly at intermediate SFC values, whereas in the latter case data were found to be significantly different from those determined by the off-line instrument.

The performance of conventional benchtop NMR and single-sided NMR applied to the study of sunflower oilin-water emulsions was investigated by Pedersen et al. [41]. With conventional NMR, the decay rate of pure water obtained after applying a CPMG sequence is much longer than that of oil, which means that the relaxation behavior of the two components is very different. With single-sided NMR, the presence of a strong magnetic field gradient heavily modifies the decay of magnetization; in fact, the decay rate of water is much faster than that of oil because water is much more affected by diffusion than oil. Nevertheless, this effect makes it possible to unequivocally distinguish the signal of water from oil. The trend in relaxation behavior with increasing oil content measured by single-sided NMR was found to be the reverse of that obtained by conventional benchtop NMR. The reverse of the trend in the decay rates with decreasing the oil content is also foreseeable as a result of the strong magnetic field gradient which affects the apparent transverse relaxation time of water due to its rapid diffusion rate. Authors demonstrated that NMR decays obtained by both conventional benchtop NMR and single-sided NMR can be readily deconvoluted in two components in the case of oil-in-water emulsions with oil content ranging from 10 to $67 \%$.

Hiaduc et al. [42] reported a feasibility study on the use of single-sided NMR for the non-invasive throughpackage' assessment of the microstructural quality of food material. Authors investigated model systems consisting of oil-in-water emulsion gels stabilized by proteins. These systems form complex structures made of fat droplets and a protein aggregate network where water is dispersed in pores with different size. An important quality parameter of such systems is the water exudation (WE). In a classical approach, WE is determined by measuring the amount of water lost from the system when subject to gravitational forces. Multivariate calibration techniques were applied to establish a relation between signals obtained by singlesided NMR and WE. Specifically, to obtain a calibration model allowing for a physical interpretation, an approach based on multilinear regression (MLR) was applied to decays collected with a benchtop instrument and with a single-sided sensor as well. Decays were transformed from the continuous domain to the discrete domain of $T_{2 \text { eff }}$ distributions and amplitudes using the non-negative least squares (NNLS) algorithm. The decays were averaged and the obtained data fitted without any initial guess on the number of components or $T_{2 \mathrm{eff}}$ values. To summarize, the MLR model was built using the NNLS amplitudes as responses, and the functional parameters as predictors. The goodness of fits of NNLS_MLR model based on conventional benchtop NMR decays (a) and decays measured with single-sided NMR (b) is observable in Fig. 3. The quality of MLR model applied to both set of data is comparable, thus indicating that single-sided NMR 

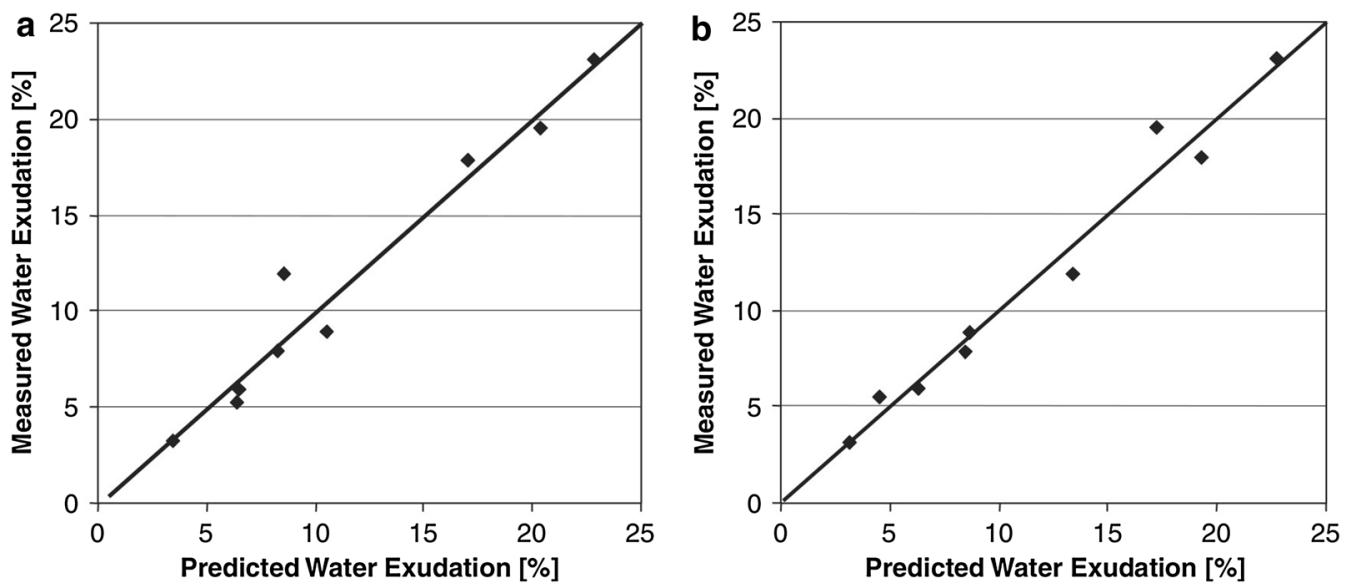

Fig. 3 Fits of NNLS-MLR models for water exudation based on (a) decays collected by a conventional benchtop NMR, and (b) by single-sided NMR. Reproduced with permission from [38]

may also allow for the assessment of WE in a through package mode. It must be taken into account that due to slow $T_{1}$-relaxation, the delay between the individual averages was set to $4 \mathrm{~s}$, which yields an experimental time of 8 min for one MOUSE measurement.

A new procedure for rapid and non-destructive determination of the fat content in dairy products has been proposed by Veliyulin et al. [28]. In this work, a novel design of unilateral NMR instrument with larger measurement volume and higher signal to noise ratio [22, 27] has allowed to reduce the time measurement of the fat content in packaged dairy products about up to few seconds. Two methods were tested: a diffusion-edited CPMG technique and a strongly $T_{1}$-weighted conventional CPMG method requiring total measurement times of about 39 and $7 \mathrm{~s}$, respectively. The latter technique has a potential for being implemented for industrial monitoring of the fat content in both packaged products and larger batches of dairy products. Linear relationship between the transverse relaxation time of the fat component and the inverse fat content was observed.

The same unilateral NMR device was tested by Veliyulin et al. [43] to set up rapid non-destructive measurement of the fat content in live or slaughtered Atlantic salmon. The total analysis time per fish was about $20 \mathrm{~s}$. The distribution of transverse relaxation times when measured with a conventional benchtop NMR instrument shows three peaks. The shortest component is usually ascribed to water closely associated with macromolecules, the intermediate one to intracellular water or water within the myofibrillar structure, whereas the longest one accounts for both lipids and water. The overlapping of the fat signal with that of extra-myofibrillar water (both contributing to the longest component) makes very difficult to differentiate fat and water content based on the quantitative $T_{2 \mathrm{eff}}$ measurements. When measurements are performed by a single-sided sensor, the effective transverse relaxation time is affected by strong field gradient generated by the sensor which influences the self-diffusion coefficient. Because the average self-diffusion coefficient of water in fish muscle is considerably faster than that of fat, this difference may be exploited to make accurate quantification of the fat component from the transverse relaxation response. Figure 4 compares the transverse relaxation curves of pure fish oil and Atlantic salmon white muscle obtained applying a CPMG sequence optimized to ensure the best separation between water and fat components. Prior to perform measurements, the instrument was calibrated using samples with known fat content (mixtures of fish oil and agarose) and characterized by NMR relaxation properties similar to those of fish fat. A significant correlation was

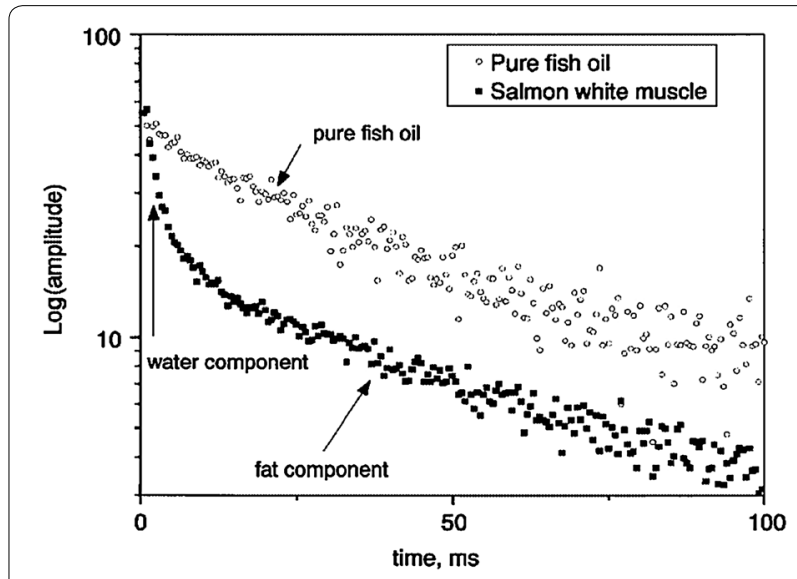

Fig. 4 Transverse relaxation curves of pure fish oil and Atlantic salmon white muscle measured at $4{ }^{\circ} \mathrm{C}$ using a single-sided NMR sensor. Reproduced with permission from [42] 
found between the fat content measured by single-sided NMR and that measured by chemical analysis. Furthermore, the mobile single-sided sensor was used to map the fat distribution over the whole fish surface. The NMR signal measured on about 50 regions was processed to obtain a post-mortem fat distribution image in the white muscle, red muscle and belly flap of Atlantic salmon.

It is well known that the application of conventional single-sided NMR to highly mobile, watery phases in foods and beverages is hampered by the strong magnetic field. In their paper, Stork et al. used both a single-sided NMR sensor with a reduced magnetic field gradient and another ("semisingle-sided") sensor design with an open sample bay for determining the oxygen content in unopened bottles with superoxygenated table water, and compared the obtained results with those from conventional NMR [44]. In the semisingle-sided sensor, the distance between the two arrays of magnet bricks was chosen in a way that 1.5-L bottles fit in the gap of the magnet system. The latter design allows much better sensitivity without sacrificing the necessary open access needed for measurements on entire food packages such as bottle. For the NMR determination of dissolved oxygen in table waters, the longitudinal relaxation time of the water was measured. It was related to the oxygen concentration $C_{\mathrm{Ox}}$ according to the following relationship:

$$
\frac{1}{T_{1}}=\frac{1}{T_{1 \mathrm{o}}}+r_{1, \mathrm{ox}} C_{\mathrm{ox}}
$$

where $T_{1 \mathrm{o}}$ denoting the relaxation time of the oxygenfree water.

As the relaxivity $r_{1, \mathrm{ox}}$ of oxygen in water is field dependent [45] and no sufficiently precise relaxation data at a proton resonance frequencies of $5-6 \mathrm{MHz}$ are available in the literature, authors performed calibration measurements against samples with a known (supersaturated) oxygen concentration.

Figure 5a shows the comparison between the spin-lattice relaxation rate measured with a single-sided NMR sensor and that measured by conventional NMR. The two sets of data were found to be in a very good agreement. The semisingle-sided sensor was also used for determining the oxygen concentration in a commercial bottle before and after opening the bottle by measuring the water relaxation time; data are reported in Fig. 5b. The sensor was capable of monitoring the oxygen concentration that was constant before opening, and progressively decreased after opening the bottle, thus indicating the possible use of this sensor for online application after a suitable calibration procedure. To achieve a better signal/noise ratio, the measuring times for each relaxation time in these curves were several hours per data point in the single-sided setup and about $40 \mathrm{~min}$ in
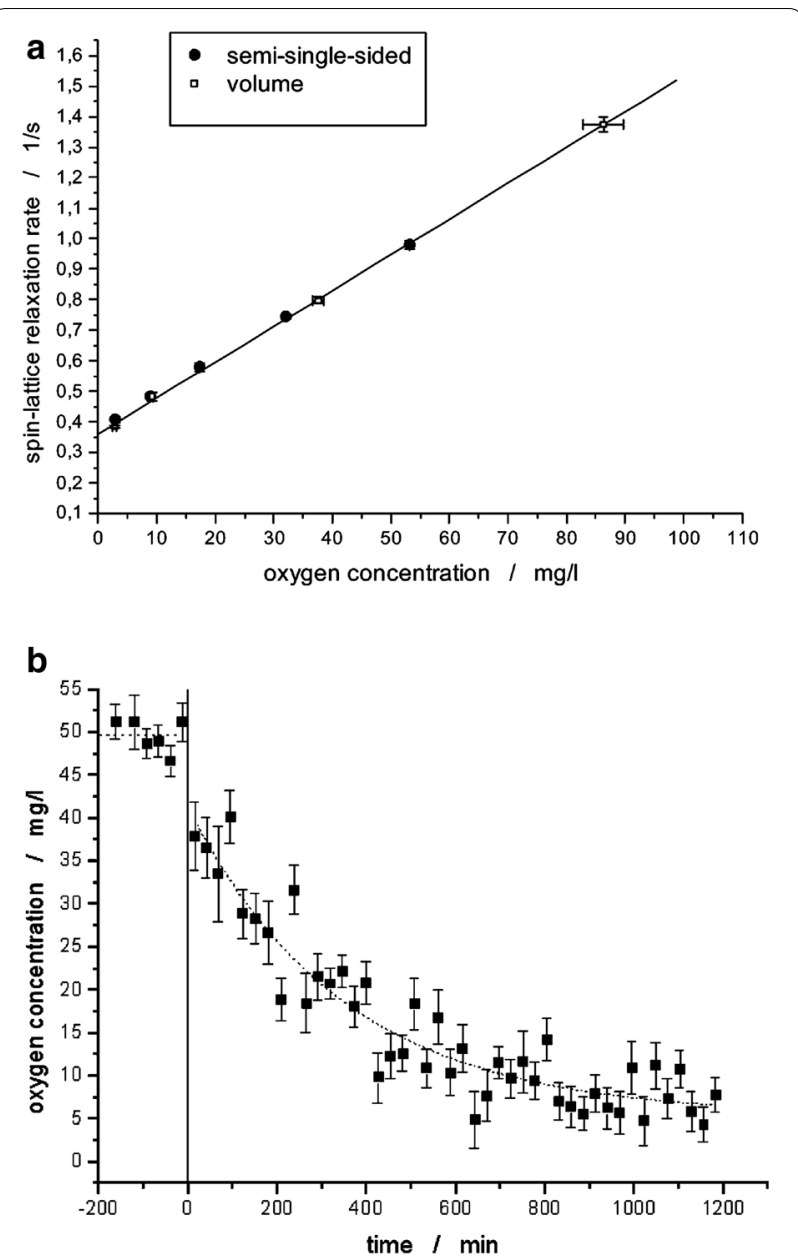

Fig. 5 a Spin lattice relaxation rates measured with a single-sided NMR sensor as compared with those obtained by a conventional NMR instrument. b Oxygen concentration measured by a semisinglesided NMR on a bottle of oxygenated table water, left of the line, before opening, right of the line, after opening (Reproduced with permission from [43])

the semisingle-sided setup. Therefore, single-sided setup imposes a measuring time about four times longer than that for semisingle-sided.

Another paper reports a method based on the use of the profile NMR MOUSE [29] to detect adulteration of virgin olive oil through sealed bottles [46]. With this method, the transverse relaxation time and the selfdiffusion coefficient of virgin olive oils adulterated with different percentages of sunflower oil and red palm oil were investigated. Authors developed a two-dimensional Laplace inversion according to the algorithm by Venkataramanan [47] to reconstruct the two-dimensional probability density distribution of the transverse relaxation time and self-diffusion coefficient. The two-dimensional $D-T_{2}$ distributions were projected to the $D$ axis and $T_{2}$ axis, as shown in Fig. 6a, b. 
In Fig. 6a, from right to left, there are four peaks representing the pure red palm oil, $10 \%$ red palm and $90 \%$ olive mixed oil, $20 \%$ red palm and $80 \%$ olive mixed oil and the pure extra virgin olive oil. The self-diffusion coefficient increases as the percentage of red palm oil increases. While in Fig. 6b, the $T_{2}$ decreases as the percentage of red palm oil increases, giving 67.5, 64.1, 60.8 and $54.9 \mathrm{~ms}$, respectively. Comparing with the pure extra virgin olive oil, the red palm mixed oil has a larger selfdiffusion coefficient but smaller $T_{2}$. These data indicate that the adulteration of extra virgin olive oils with sunflower oil may be readily detected from the self-diffusion coefficient behavior, whereas the adulteration with red palm oil can be detected from both diffusion and transverse relaxation behavior.

The tomato paste processing industry is very interested in developing methods to detect tomato spoilage in 1000-L non-ferrous, metal-lined containers without violating the seal. An early spoilage detection would eliminate shipping costs and disposal costs when spoiled tomato paste arrives at destination. Pinter et al. [48] explored the relaxation
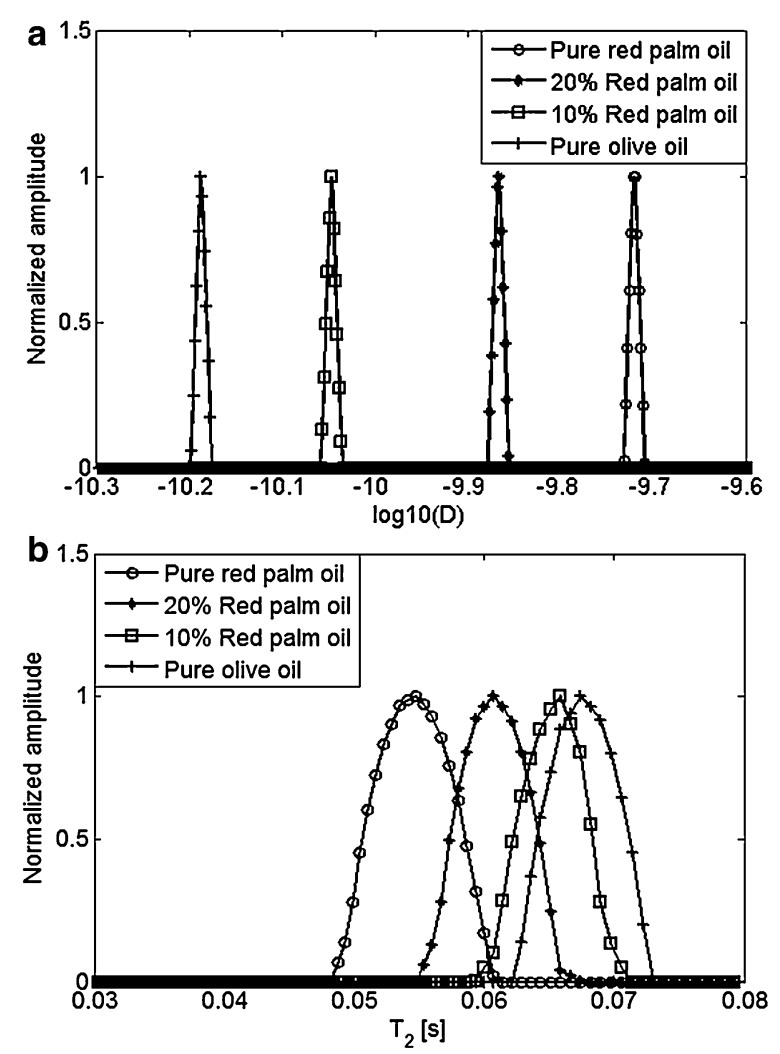

Fig. 6 D-T distribution maps of oils. From the top to the bottom (a) pure extra virgin olive oil, extra virgin olive oil adulterated with 10 and $20 \%$ of sunflower oil, pure sunflower oil. b Pure red palm oil, extra virgin olive oil adulterated with 10 and $20 \%$ of red palm oil, and pure extra virgin olive oil (Reproduced with permission from [45]) properties of sterile and unsterile tomato paste samples as a function of time at both low and room temperature. As long as the storage temperature was maintained below $5^{\circ} \mathrm{C}, T_{1}$ and $T_{2}$ values remain constant over the course of at least 1 month, suggesting that the low temperature significantly slows the bacteria-mediated tomato paste spoilage. At room temperature where tomato processing, packaging and storage occur, the relaxation times for sterile and unsterile samples were different. Although the room temperature $T_{2}$ value was constant as a function of time for these samples, the corresponding $T_{1}$ value increased exponentially in the first days and tended to a limit value, indicated as $T_{1}^{\infty}$, for long time of observations. Specifically, the steady-state limit value of spin lattice relaxation time $T_{1}^{\infty}$ obtained by the regression of $T_{1}$ values measured as a function of time was the parameter chosen by authors to differentiate between spoiled and unspoiled tomato paste. This result prompted them to extend the study to $1000-\mathrm{L}$ non-ferrous, metal-lined totes using single-sided NMR. To perform measurements directly on the metal container,
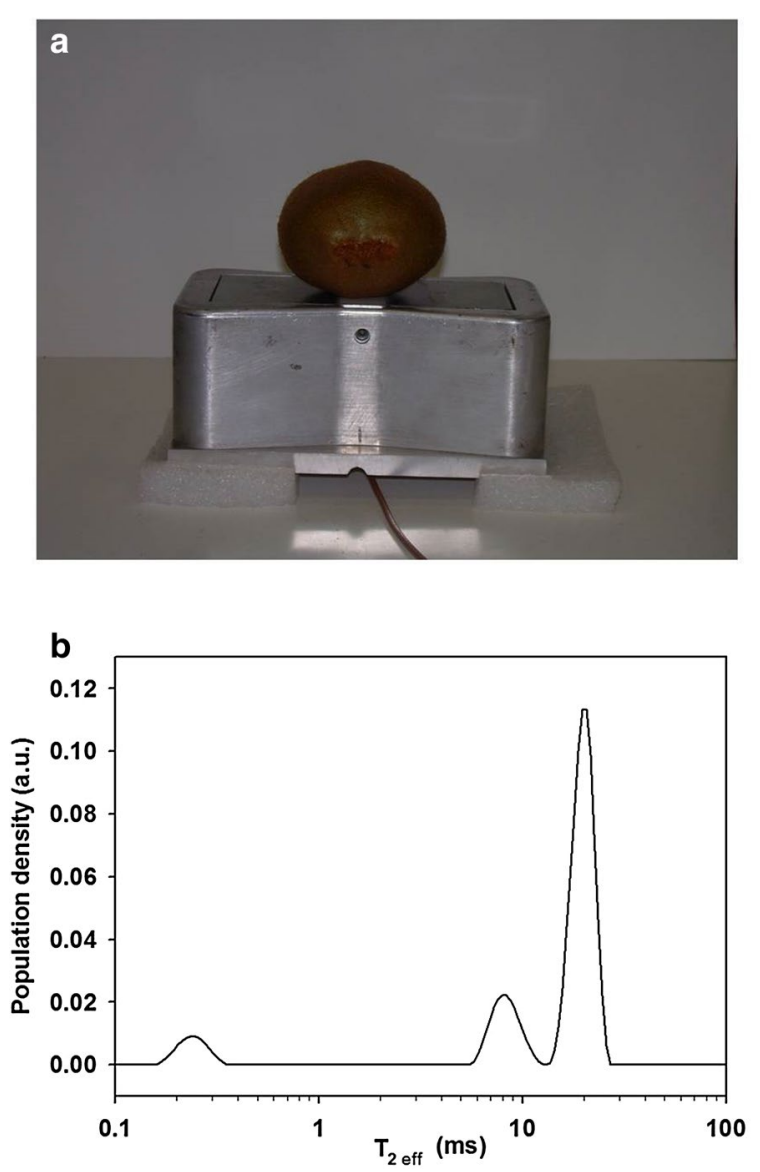

Fig. 7 a Measurement of intact kiwifruit with a single-sided NMR sensor. b Transverse relaxation times distribution measured on a ripened kiwifruit 
its effect on the circuit tuning and impedance matching properties was properly compensated. A modified saturation recovery sequence was used to measure the longitudinal relaxation with single-sided NMR. The NMR signal and $T_{1}$ values obtained from the large format container with the single-sided sensor suggested that this device can be used to study tomato paste spoilage in factory process environments.
Capitani et al. monitored the water status of kiwifruits as a function of season using a single-sided NMR sensor $[49,50]$. Using this sensor, it was possible to measure the entire fruit at a depth of about $0.5 \mathrm{~cm}$ from the peel surface without cutting it, see Fig. $7 \mathrm{a}$. The $T_{2 \mathrm{eff}}$ distribution of a mature kiwifruit measured by single-sided NMR shows three peaks, see Fig. 7b. According to the literature, the longest $T_{2 \text { eff }}$ component was assigned to
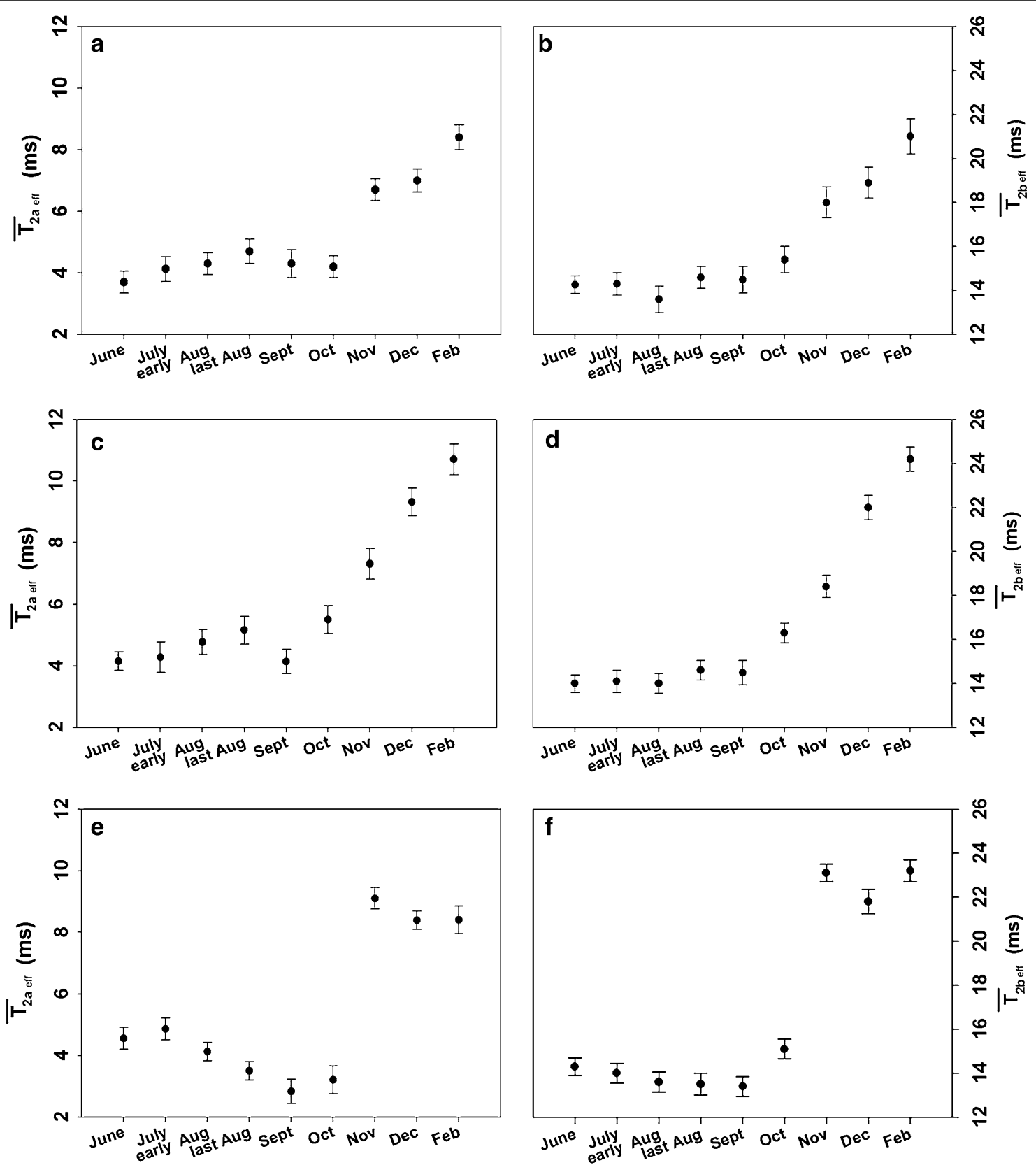

Fig. 8 Average $T_{2}$ values for the intermediate $\left(\bar{T}_{2 a}\right)$ and the longest $\left(\bar{T}_{2 b}\right)$ components measured on Hayward (a, b), Cl.GI (c, d), and Zespri (e, f) kiwifruits as a function of their developmental stage (Reproduced with permission from [49]) 
protons in vacuole, the intermediate one to proton in cytoplasm and extra-cellular space, and the shortest one to proton in cell walls. It must be noted that the presence of the strong magnetic field gradient of the single-sided sensor heavily shortens $T_{2 \text { eff }}$ values. In fact in the case of ripened kiwifruit measured in homogeneous fields, literature data report average $T_{2 \mathrm{eff}}$ values of $800-1000 \mathrm{~ms}$ for the longest component, $200-400 \mathrm{~ms}$ for the intermediate component, and $20-80 \mathrm{~ms}$ for the shortest one. With single-sided NMR, the longest component is as short as $20 \mathrm{~ms}$. Nevertheless, single-sided NMR was suitable to monitor the growth of kiwifruit. Because the shortest component was very poorly affected by the season, only the intermediate and longest components were taken into account. Figure 8 reports the average values of the intermediate component ( $\left.\bar{T}_{2 a \text { eff }}\right)$ and the longest component $\left(\bar{T}_{2 b \text { eff }}\right)$ measured on nine kiwifruits of three cultivar,

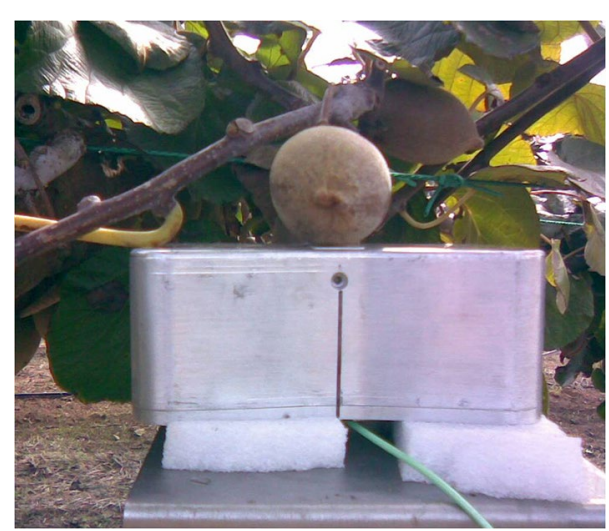

Fig. 9 NMR measurements in field on entire kiwifruits attached to the plant (Reproduced with permission from [49]) namely Hayward (a, b), CI.GI (c, d), and Zespri (e, f) at different stages of development. In all cultivars, $T_{2 \text { eff }}$ values were found to be rather constant until October, thereafter they increased. The tendency toward longer $T_{2 \text { eff }}$ relaxation times later in the season is consistent with a change in the fruit texture occurring during fruit development. However, whereas in Hayward and CI.GI a gradual lengthening of $T_{2 \mathrm{eff}}$ was observed, in Zespri, a net and sharp lengthening of both components occurred between October and November, indicating the earlier maturation of Zespri.

The ripening of kiwifruits was also monitored in field on fruits attached to the plant during three campaigns of measurement carried out in October, November and December, see Fig. 9. Figure 10 shows the transverse relaxation decays measured in field. A preliminary optimization of conditions was necessary to achieve a good signal to noise ratio and minimize the measuring times (within $8 \mathrm{~min}$ ). In all cultivars, a lengthening of the decays with the season was observed; however, the process was complete already in November for Zespri as shown by the perfect overlapping of decays measured in November and December. In agreement with data collected in laboratory on intact kiwifruits, these data confirmed the earlier maturation of Zespri.

The profile NMR MOUSE was used to get preliminary results on three fresh intact blueberries (belonging to the same cultivar) and evaluate the effects of withering outside the fridge for 3 and 6 days [51], see Fig. 11. With this sensor NMR depth profiles were collected, these profiles encode the amplitude of the ${ }^{1} \mathrm{H}$ NMR signal as a function of the depth scanned. The amplitude of profiles measured on withered blueberries was found to be
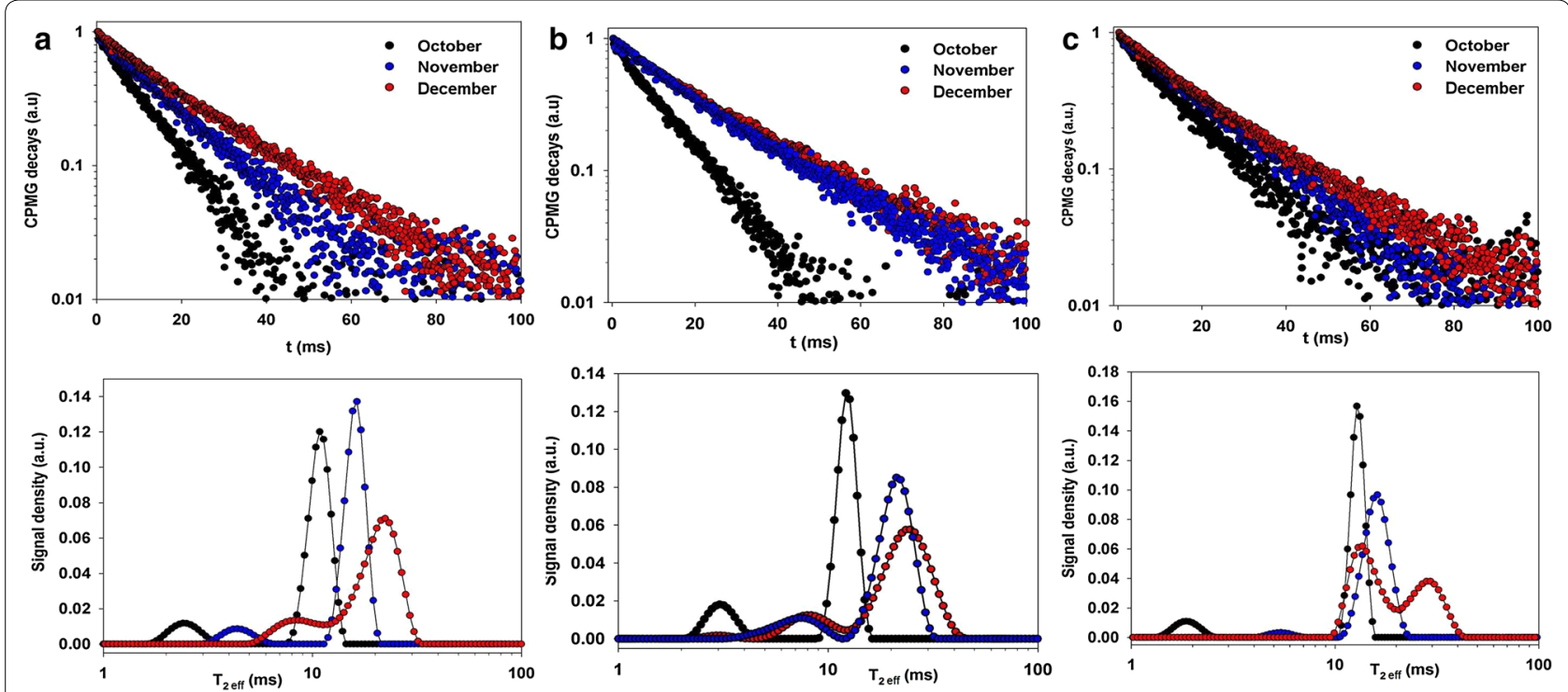

Fig. 10 Transverse relaxation curves measured in field on Hayward (a), Cl.GI (b), and Zespri (c) with the corresponding relaxation times distributions 


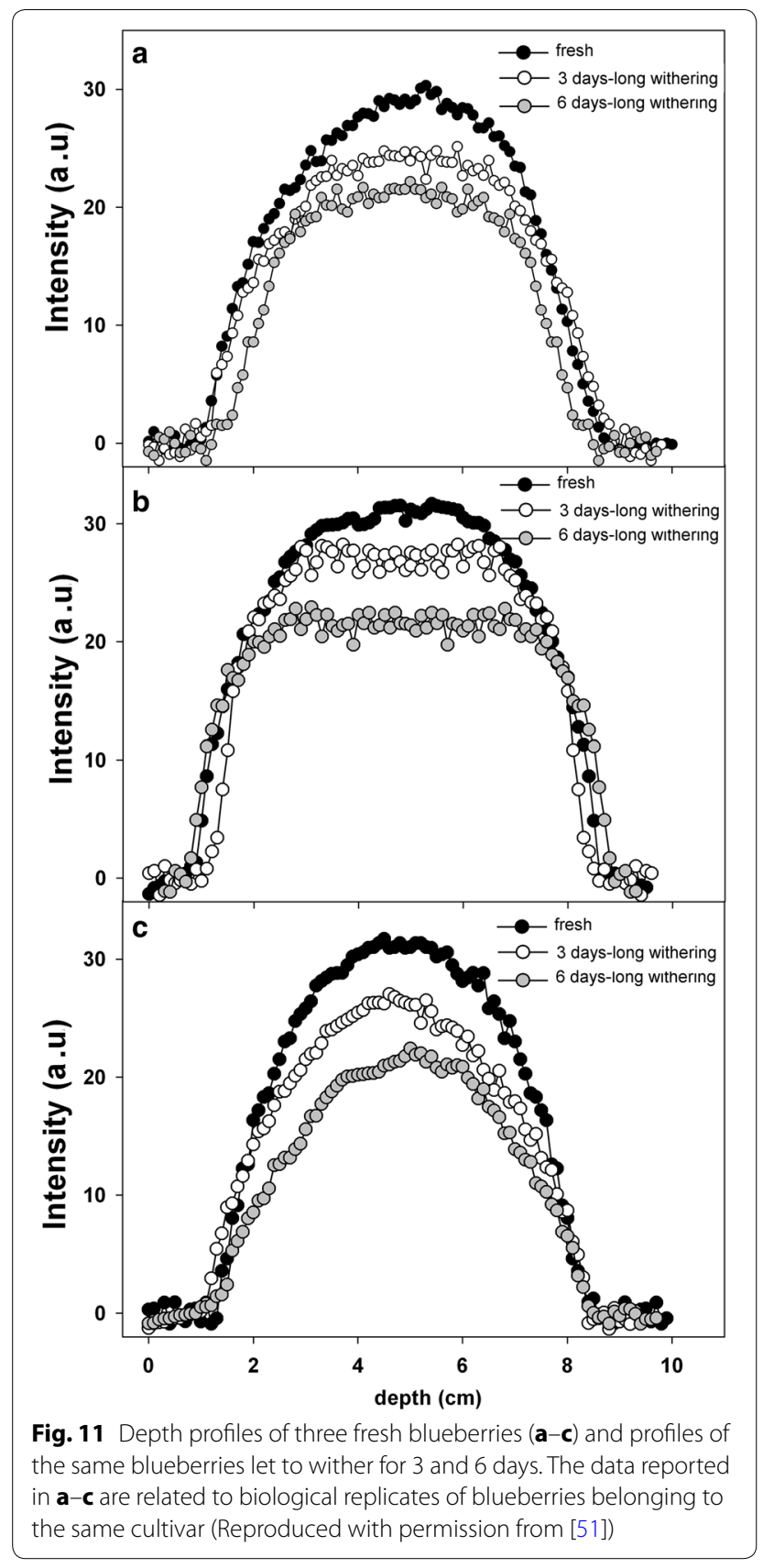

lower than that measured on fresh blueberries, indicating a loss of water that was quantified by integrating the profiles. After 3 days of withering, a loss of water of $13 \%$ (a), $11 \%$ (b), and $16 \%$ (c) was measured, whereas after 6 days the loss of water was found to be $30 \%$ (a), $20 \%$ (b), and $34 \%$ (c). Therefore, the integral of the profile is a suitable index of water loss. The same index may be used to monitor changes in foodstuff texture due to maturation, ripening, water and osmotic stress, for monitoring the effect of different types of processing on food matrices, and the effect of storage.
Adiletta et al. used the profile NMR MOUSE to investigate the drying process on pears [52]. Drying is an important process for conservation and marketing of fruits due to their high water activity which makes them perishable. Therefore, the knowledge and optimization of drying process are very important to minimize thermal damage and quality loss. Information on drying kinetics was obtained by measuring the intensity of ${ }^{1} \mathrm{H}$ NMR signal as a function of the thickness of the sample. Figure 12 shows the comparison among profiles of fresh pear and pears dried for 3, 6, 15, 20, 29, and $48 \mathrm{~h}$. The amplitude of profiles measured in dried samples progressively lowered and also the thickness of the profiles progressively decreased, thus indicating a loss of water with increasing of drying time which was accompanied by shrinkage. An evaluation of the loss of water in the outer, intermediate and central regions of pears samples was obtained simply by integrating the profiles in three corresponding regions (without cutting the samples); the results obtained are reported in Fig. 13a. As expected, the water content of the outer region decreases more quickly than that of the intermediate and central ones. Such differences become less pronounced at relatively long drying times. Figure $13 \mathrm{~b}$ shows the good agreement between values of moisture content $\left(M_{t} / M_{0}\right)$, obtained by gravimetric method and values of hydrogen content $\left(I_{t} / I_{0}\right)$ obtained by integrating NMR depth profiles, with a regression coefficient of 0.978 .

\section{Concluding remarks}

Research applications described above illustrate that unilateral NMR sensors present an attractive option for non-invasive assessment of compositional and microstructure of food materials. The first applications of the unilateral NMR devices in foods were aimed at measuring the water/oil contents in food emulsions and to determine solid fat content. Successively their use has been extended to other foodstuffs as well as at packaged foods. Several applications focused on assessment of food quality and monitoring processes such as ripening, growth rate of fruits, drying process, and so on. The fact that single-sided NMR devices allow easy sample access and portability makes them attractive for quality and process control in the food industry. A main handicap of these devices is magnet field inhomogeneity, which significantly compromises sensitivity and robustness under practical circumstances. The use of echo techniques is typically necessary since FIDs obtained by such devices are also compromised by very short $T_{2 \text { eff }}$ values. Other unilateral magnets have been developed with more homogeneous magnetic field, larger sensitive volume and higher sensitivity. These sensors allowed to shorten 


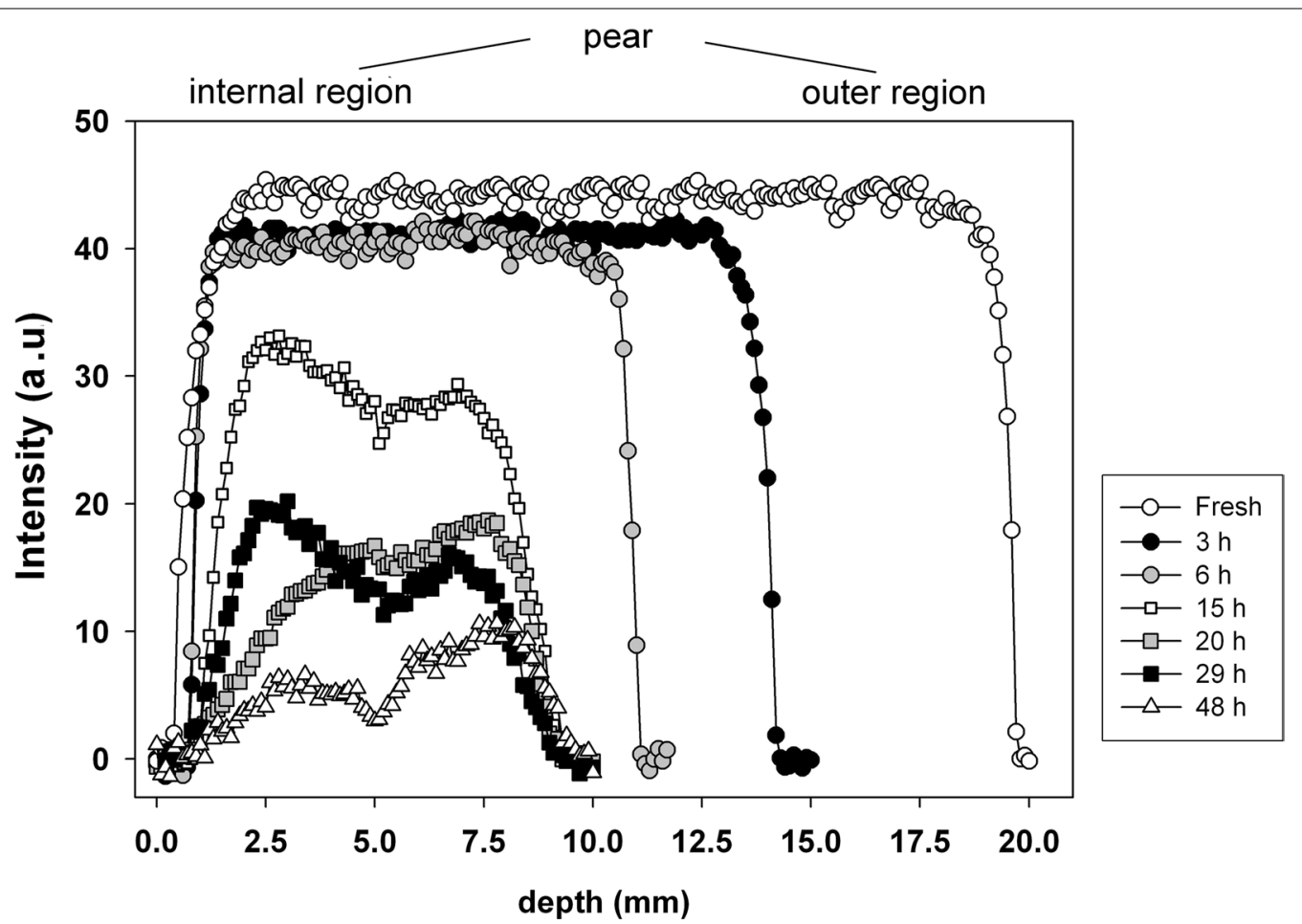

Fig. 12 NMR depth profiles of fresh pear and pear samples dried at $50^{\circ} \mathrm{C}$ for 3, 6, 15, 20, 29 and $48 \mathrm{~h}$ (Reproduced with permission from [51])
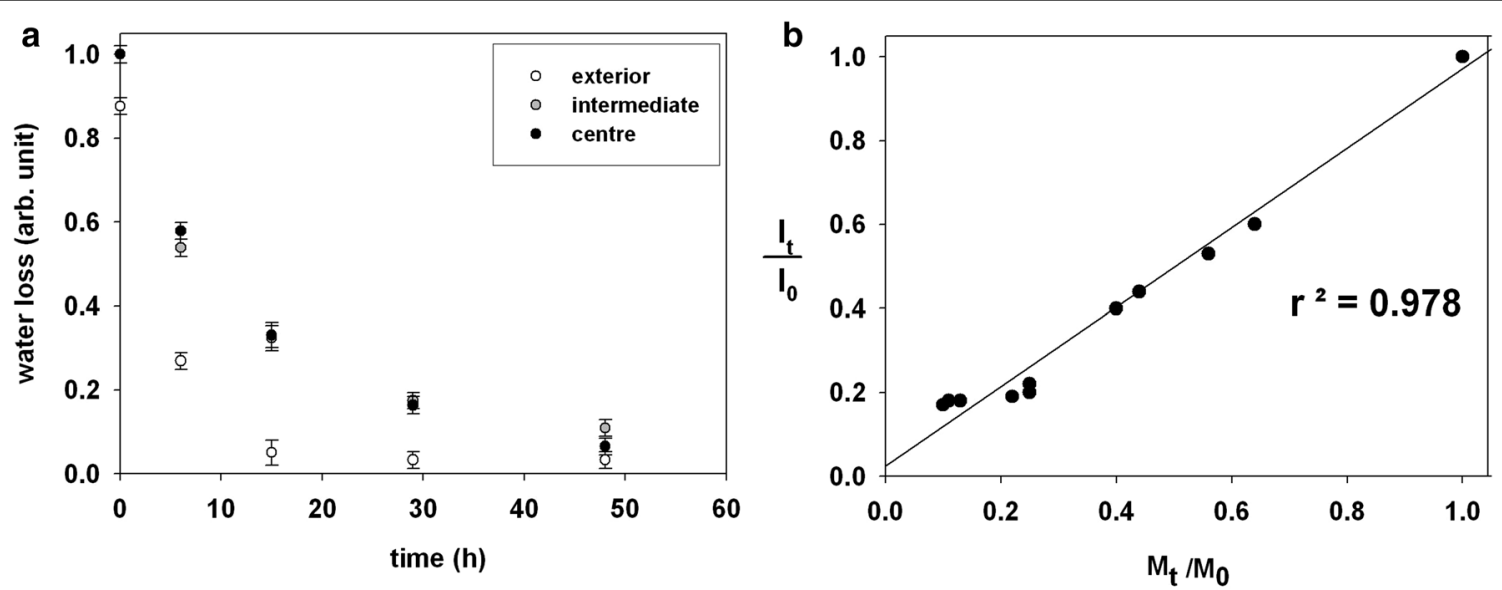

Fig. 13 a Water loss in exterior, intermediate, and central part of pear samples measured by single-sided NMR. $\mathbf{b}$ Relationship between the water loss measured by gravimetric method $\left(M_{t} / M_{0}\right)$ and single-sided NMR $\left(I_{t} / I_{0}\right)$ (Reproduced with permission from [51])

measurement times thus paving the way to their future employment in a quality control context.

Nevertheless, use of unilateral NMR devices is still limited. Further studies are needed to expand their utilization to other food matrices and develop protocols to be applied in online inspections for monitoring quality and food processes.
Authors' contributions

All authors contributed equally to this review. All authors read and approved the final manuscript.

\section{Author details}

${ }^{1}$ Istituto di Metodologie Chimiche, Laboratorio di Risonanza Magnetica, "Annalaura Segre", CNR, Monterotondo, 00015 Rome, Italy. ${ }^{2}$ Dipartimento di Chimica e Tecnologie del Farmaco, Sapienza Università di Roma, Piazzale Aldo Moro 5, 00185 Rome, Italy. 


\section{Acknowledgements}

This work was supported by "e-ALIERB: un OPEN LAB per caratterizzare e valorizzare i prodotti alimentari ed erboristici del territorio laziale" Project (Regione Lazio LR13/2008).

\section{Competing interests}

The authors declare that they have no competing interests.

\section{Funding}

This work was supported by "e-ALIERB: un OPEN LAB per caratterizzare e valorizzare i prodotti alimentari ed erboristici del territorio laziale" Project (Regione Lazio LR13/2008).

\section{Publisher's Note}

Springer Nature remains neutral with regard to jurisdictional claims in published maps and institutional affiliations.

Received: 4 November 2016 Accepted: 24 March 2017

Published online: 17 July 2017

\section{References}

1. van Duynhoven J, Voda A, Witek M, van As H. Time domain NMR applied to food products. Annu Rep NMR Spectrosc. 2010;69:145-97.

2. Hills BP. Applications of low field NMR to food science. Annu Rep NMR Spectrosc. 2006;58:179.

3. Hills BP, Clark CJ. Quality assessment of horticultural products by NMR. Annu Rep NMR Spectrosc. 2003;50:75.

4. Belton PS. Using magnetic resonance to explore food microstructures in food microstructures microscopy, measurements and modelling. In: Morris VJ, Groves K, editors. Cambridge: Woodhead Publishing.

5. Tylewicz U, Panarese V, Laghi L, Rocculi P, Nowacka M, Placucci G, Dalla Rosa M. NMR and DSC water study during osmotic dehydration of Actinidia deliciosa and Actinidia chinensis Kiwifruit. Food Biophys. 2011;6:327-33.

6. Santagapita P, Laghi L, Panarese V, Tylewicz U, Rocculi P, DallaRosa M. Modification of transverse NMR relaxation times and water diffusion coefficients of kiwifruit pericarp tissue subjected to osmotic dehydration. Food Bioprocess Technol. 2013;6:1434-43.

7. Hills BP, Remigereau B. NMR studies of changes in subcellular water compartmentation in parenchyma apple tissue during drying and freezing. Int J Food Sci Technol. 1997;32:51-61.

8. Marigheto M, Venturi L, Hills BP. Postharvest Biol Technol. 2008:48:331-40.

9. Hills BP. NMR relaxation and diffusion studies of horticultural products in modern magnetic resonance. Netherlands: Springer; 2008. p. 1721-7.

10. Barreiro P, Moya A, Correa E, Ruiz-Altisent M, Fernandez-Valle M, Peirs A, Wright KM, Hills BP. Appl Magn Reson. 2002;22:387.

11. Hills BP, Nott KP. NMR studies of water compartmentation in carrot parenchyma tissue during drying and freezing. Appl Magn Reson. 1999;17:521-35.

12. Marigheto N, Vial A, Wright K, Hills B. A combined NMR and microstructural study of the effect of high-pressure processing on strawberries. Appl Magn Reson. 2004;26:521-31.

13. Marques JPM, Rutledge DN, Ducauze CJ. Low resolution pulse nuclear magnetic resonance study of water equilibration in dried carrots. Int J Food Sci Technol. 1991;26:173.

14. Keener KM, Stroshine RL, Nyenhuis JA.J Am Soc Hortic Sci. 1999:124:289.

15. Cho BK, Chayaprasert W, Stroshine RL. Postharvest Biol Technol. 2008:47:81.

16. Brown RJ, Capozzi F, Cavani C, Cremonini MA, Petracci M, Placucci G. Relationships between ${ }^{1} \mathrm{H}$ NMR relaxation data and some technological parameters of meat: a chemometric approach. J Magn Reson. 2000;147:89-94.

17. Bertram HC, Schafer A, Rosenvold K, Andersen HJ. Physical changes of significance for early post mortem water distribution in porcine $M$. longissimus. Meat Sci. 2004:66:915.

18. Bertram HC, Kristensen M, Andersen HJ. Functionality of myofibrillar proteins as affected by $\mathrm{pH}$, ionic strength and heat treatment-a low-field NMR study. Meat Sci. 2004;68:249.
19. Todt H, Guthausen G, BurkW, Schmalbein D, Kamlowski W. Water/moisture and fat analysis by time-domain NMR. Food Chem. 2006;96:436-40.

20. Zalesskiy SS, Danieli E, Blümich B, Ananikov VP. Miniaturization of NMR systems: desktop spectrometers, microcoil spectroscopy, and "NMR on a chip" for chemistry, biochemistry, and industry. Chem Rev. 2014;114:5641-94.

21. Blümich B. Introduction to compact NMR: a review of methods. Trends Anal Chem. 2016;83:2-11

22. Marble AE, Mastikhin IV, Colpitts BG, Balcom BJ. A compact permanent magnet array with a remote homogeneous field. J Magn Reson. 2007;186:100-4.

23. Manz B, Coy A, Dykstra R, Eccles CD, Hunter MW, Parkinson BJ, Callaghan PT. J Magn Reson. 2006;183:25-31.

24. Blümich B, Perlo J, Casanova F. Mobile single-side NMR. Progr Nucl Magn Reson Spectrosc. 2008;52:197-269.

25. Eidmann G, Savelsberg R, Blumler P, Blu"mich B. The NMR mMOUSE: a mobile universal surface explorer. J Magn Reson. 1996;A122:104-9.

26. Blumich B, Anferova S, Sharma S, Segre AL, Federici C. Degradation of historical paper: nondestructive analysis by the NMR-MOUSE. J Magn Reson. 2003:161:204-9.

27. Marble AE, Mastikhin IV, Colpitts BG, Balcom BJ. An analytical methodology for magnetic field control in unilateral NMR. J Magn Reson. 2005;174:78-87.

28. Veliyulin E, Mastikhin IV, Marble AE, Balcom BJ. Rapid determination of the fat content in packaged dairy products by unilateral NMR. J Sci Food Agric. 2008;88:2563-7.

29. Perlo J, Casanova F, Blümich B. Profiles with microscopic resolution by single-sided NMR. J Magn Reson. 2005;176:64-70.

30. Goelman G, Prammer MG. The CPMG pulse sequence in strong magnetic field gradients with applications to oil-well logging. J Magn Reson. 1995;113:11-8

31. Hurlimann MD, Griffin DD. Spin dynamics of Carr-Purcell-Meiboom-Gilllike sequences in grossly inhomogeneous B0 and B1 fields and application to NMR well logging. J Magn Reson. 2000;143:120-53.

32. Hurlimann MD. Diffusion and relaxation effects in general stray field NMR experiments. J Magn Reson. 2001;148:367-78.

33. Blümich B, Haber-Pohlmeier S, Zia W. Compact NMR. Walter de Gruyter; 2014

34. Anferova S, Anferov V, Adams M, Fechete R, Schroeder G, Blümich B. Thermo-oxidative aging of elastomers: a temperature control unit for operation with the NMR-MOUSE. Appl Magn Reson. 2004;27(3):361-70.

35. Mitchell J, Gladden LF, Chandrasekera TC, Fordham EJ. Low-field permanent magnets for industrial process and quality control. Progr Nucl Magn Reson Spectrosc. 2014;76:1-60.

36. Guthausen A, Guthausen G, Kamlowski A, Todt H, Burk W, Schmalbein D. Measurement of fat content of food with single-sided NMR. JAOCS. 2004:81:727-31.

37. Guthausen G, Todt H, Burk W, Schmalbein D, Guthausen A, Kamloski A. Single sided NMR in food. In: Modern magnetic resonance. Berlin: Springer; 2006. p.1895-7.

38. Vandeginste RGM, Massart DL, Buydens LMC, De Jong S, Lewi PJ, Smeyers-Verbeke J. Handbook of chemometrics and qualimetrics. Amsterdam: Elsevier Science Publisher; 1998.

39. Guthausen G. Analysis of food and emulsions. Trends Anal Chem. 2016;83:103-6.

40. Martini S, Herrera ML, Marangoni A. New Technologies to determine solid fat content on-line. JAOCS. 2005:82:313-3179.

41. Pedersen HT, Ablett S, Martin DR, Mallet MJD, Engelsen SB. Application of the NMR-MOUSE to food emulsions. J Magn Reson. 2003;165:49-58

42. Haiduc AM, Trezza EE, van Dusschoten D, Reszka AA, van Duynhoven JPM. Non-invasive through-package assessment of the microstructural quality of a model food emulsion by the NMR MOUSE. Food Sci Technol. 2007:40:737-43.

43. Veliyulin E, van der Zwaag C, Burk W, Erikson U. In vivo determination of fat content in Atlantic salmon (Salmo salar) with a mobile NMR spectrometer. J Sci Food Agric. 2005:85:1299-304

44. Stork H, Gädke A, Nestle N. Single-sided and semisingle-sided NMR sensors for highly diffusive samples: application to bottled beverages. JAFC. 2006:54:5247-55252.

45. Teng CL, Hong H, Kühne S, Bryant RG. Molecular oxygen spin-lattice relaxation in solutions measured by proton magnetic relaxation dispersion. J Magn Reson. 2001;148:31-4. 
46. Xu Z, Morris RH, Bencsik M, Newton MI. Detection of virgin olive oil adulteration using low field unilateral NMR. Sensors. 2014;14:2028-35.

47. Venkataramanan L, Song YQ, Hurlimann MD. Solving Fredholm integrals of the first kind with tensor product structure in 2 and 2.5 dimensions. IEEE Trans Sign Process. 2002;50:1017-26.

48. Pinter MD, Harter T, McCarthy MJ, Augustine MP. Towards using NMR to screen for spoiled tomatoes store in 1,000 L aseptically sealed. MetalLined Totes Sensors. 2014;14:4167-76.

49. Capitani D, Mannina L, Proietti N, Sobolev AP, Tomassini A, Miccheli A, Di Cocco ME, Capuani G, De Salvador FR. Monitoring of metabolic profiling and water status of Hayward kiwifruits by nuclear magnetic resonance. Talanta. 2010;82:1826-38.
50. Capitani D, Mannina L, Proietti N, Sobolev AP, Tomassini A, Di Cocco ME, Capuani G, De Salvador FR, Delfini M. metabolic profiling and outer pericarp water state in Zespri, CIGI, and Hayward Kiwifruits. JAFC. 2013;61:1727-40

51. Capitani D, Sobolev AP, Delfini M, Vista S, Antiochia R, Proietti N, Bubici S, Ferrante G, Carradori S, De Salvador FR, Mannina L. NMR methodologies in the analysis of blueberries. Electrophoresis. 2014;35:1615-26.

52. Adiletta G, Russo P, Proietti N, Capitani D, Mannina L, Crescitelli A, Di Matteo M. Characterization of pears during drying by conventional technique and portable non invasive NMR. Chem Eng Trans. 2015;44:151-6.

\section{Submit your manuscript to a SpringerOpen ${ }^{\circ}$ journal and benefit from:}

- Convenient online submission

- Rigorous peer review

- Immediate publication on acceptance

- Open access: articles freely available online

- High visibility within the field

- Retaining the copyright to your article 